\title{
DEVELOPMENT OF RESEARCH ENGINE CONTROL UNIT USING FPGA -BASED EMBEDDED CONTROL SYSTEM
}

\author{
B. Prem Anand, C.G. Saravanan \\ Annamalai University \\ Faculty of Engineering and Technology \\ Department of Mechanical Engineering \\ Annamalainagar - 608 002, Tamilnadu, India \\ tel.: +919843678233, +919843064772, fax: +04144238275 \\ e-mail:prem.au2005@gmail.com,rsdk66@yahoo.com
}

\begin{abstract}
Though the Electronic Control Unit (ECU) systems are wide use in modern vehicles, they are manufactured by a few companies and supplied to engine manufacturers. Automotives with factory made ECUs designed to maximize the performance while minimize cost. However, factory made ECUs (FECU) have closed software and hardware. The control gain, lookup tables, and constants used in the algorithms are also calibrated for a particular engine design. Unlike the carburettor, these systems cannot be opened and studied under different operating conditions to determine their characteristics and how they influence the performance of an engine. They can be used as black boxes and work only at specified modes as determined by their electronic control unit (ECU) and more over the algorithms and their implementation are maintained as trade secret by the developers. In the research work, it often becomes necessary to study parameters like injection duration, injection timing, sensor responses etc. to ascertain their effects. Thus in such cases the factory made ECU supplied with the injection system cannot be used. The required need is fulfilled by developing a customized electronic controller that would be suitable for studying the variations at engine operating points. The project involved the development of a research ECU (RECU) using reconfigurable field programmable gate arrays (FPGA) based embedded control system for a four-stroke port fuel injected gasoline engine. A calibrated fuel map is generated for various operating points of the engine using virtual instrumentation (VI) based software while simulating functions of sensors. From the mapping, the fuel injection time and fuel delivery quantity is estimated for the required running conditions of the engine. The developed Research Electronic Control Unit (RECU) could reveal gasoline engine performance in particular for research-based applications as it involves frequent programme development and control implementations.
\end{abstract}

Keywords: ECU, RECU, engine control algorithm, labview, virtual instrumentation, FPGA, fuel map, automotive sensor

\section{Introduction}

With the market competition becoming more intense and fiercer, it is imperative to shorten the development cycle and reduce the development cost. At that instance, it is often require developing of new programmes and implementing on the test system for measurement and control application. In such cases, there is a need to conduct programmatic communication over the network ports and built in web (HTTP) and file (FTP) servers as well as to add expansion and distributed input and output $(\mathrm{I} / \mathrm{O})$ to the system. At acceleration automotive engines revolutions in the range of speed to15,000 rpm. At this speed, there is less than $4 \mathrm{~ms}$ per crankshaft rotation and the system must precisely control fuel and spark events in the angle domain to less than 1degree. The chosen approach to this problems depended heavily on high-speed processing source such as field programmable gate arrays (FPGA). An FPGA is a chip that consists of unconfigured logic gates. Unlike the fixed, vendor - defined functionality of an application - specific integrated circuit (ASIC) chip, an FPGA can be configured and reconfigured for different applications. FPGAs are used in applications where the cost of developing and fabricating an ASIC is prohibitive or the hardware must be reconfigured after being placed into service. FPGAs appear in devices such as 
electronic instruments, consumer electronics, automobiles, aircraft, copy machines, and application specific computer hardware [1-3]. FPGAs can be used to implement custom algorithms in hardware because they offer benefits over processor such as precise time and synchronization, rapid decision making and simultaneous execution of parallel tasks. However, floating-point processors have a computational advantage since FPGAs are limited to integer math. This limitation can be partially overcome by using high-level development tools, code, or IP cores that convert floating-point calculations to integer math. However, a combined FPGA/processor architecture can be used in control applications to take advantage of the strengths of each device [4-7]. This project involves developing fuel injection map while simulating function of sensors and for tracking the angular position of crankshaft using crank angle encoder and generating precise angle-based fuel and spark commands. Apart from that, sensor design has been done for acquiring online data, which would be used for developing control algorithms. These tools provide a seamless path from prototype to production when FPGA-based controllers are used for embedded - power train applications.

\section{Configuring Hardware system}

This project involved development of engine control algorithms as well as sensor design. A National instrument (NI) compactRIO (cRIO) 9074 as shown in the Fig. 1, has been chosen as chassis for the embedded control system because of its flexibility, small size and rugged form factor. With this system, sensors and actuators can be connected and the online data can be visualized in the monitor. The unique computational feature of the compact RIO system is that it includes both a real-time processor and FPGA. Both devices are programmable using the labview graphical development environment. With this combined architecture, multiple control approaches and algorithms can be quickly designed and tested on the application system.

a)

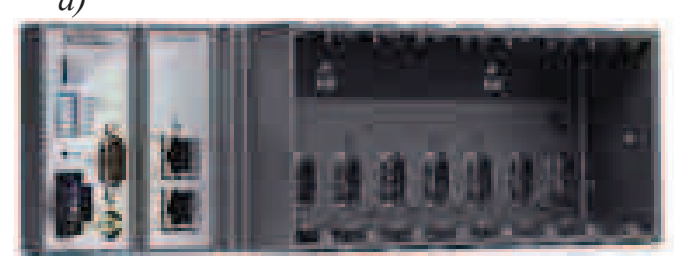

b)

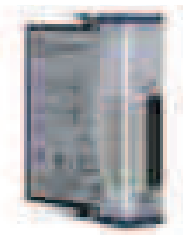

c)

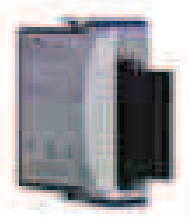

d)

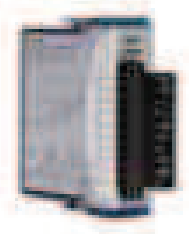

Fig. 1. a) A 9074 CompactRIO Real-Time Controller with dual Ethernet ports, b) NI 94018 Channel, 5 V/TTL HighSpeed Bidirectional C Series Digital I/O Module, c) NI 92018 Channel, \pm 10 V, 500 kS/s, 12-Bit C Series Analog Input Module, d) NI 94748 Channel, 5 to 30 V, 1 us Sourcing C Series Digital Output Module

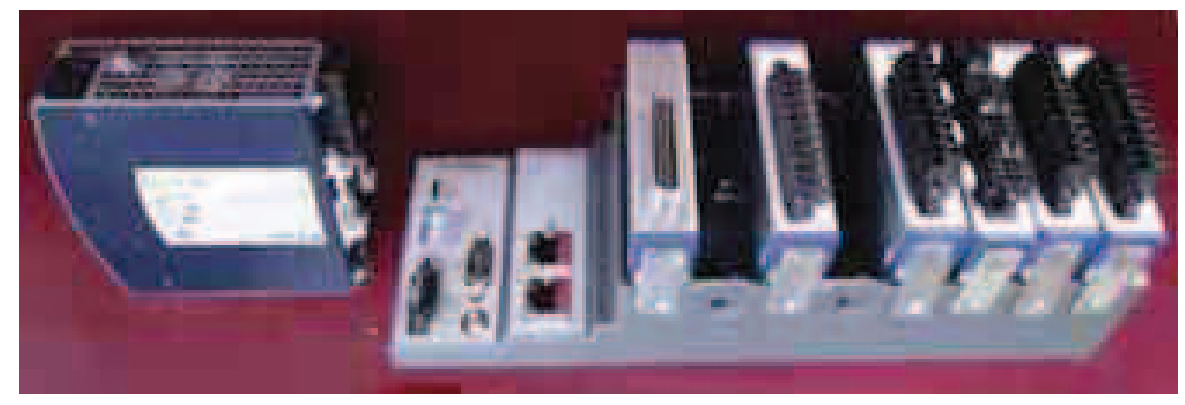

Fig. 2. NI 9074 CompactRIO Real-Time Controller with input and output modules

NI has developed a family of analog and digital input and output (I/O) modules with onboard signal conditioning and breakout connectors for the compactRIO system. Some applications, however, may require specialized modules that are not included in the NI product offering. For this case, NI provides a cRIO module development kit with the software, hardware and documentation required to create custom modules. The Research Engine Control (RCEU) unit has required 
creating three custom cRIO I/O modules with FPGA based reconfigurable embedded control system. The first module NI 9401 is an 8 - channel, $100 \mathrm{~ns}$ bidirectional compatible with 5V/TTL digital input signal generation. The developer can configure the direction of input or output digital lines by connecting a 4-bit nipple on the module. This module could be used for implementing custom, high-speed counters/timers, digital communications protocols, pulse generation and much more. The NI 9401 is connected with crank angle encoder intended to receive signal in the form of pulse for further processing. The second module NI 9201 is a C series 8 - channel, 12 bit, compatible with receiving $10 \mathrm{~V}$ analog input at a maximum aggregate rate of $500 \mathrm{Ks} / \mathrm{s}$. This module receives signal in the form of voltage range between $0-5$ which could be used for further computations. The analog input module NI 9201 is connected with Throttle Position Sensor (TPS), Manifold Absolute Position (MAP) sensor and oxygen sensor. The third module NI 9474 is C series 8 - channel, $1 \mu \mathrm{s}$ high speed sourcing, compatible with generating 5 to $30 \mathrm{~V}$ digital output. Each channel of NI 9474 has an LED that indicates the state of the selected channel functioning. This module can be directly connected to a variety of industrial devices such as motors, actuators, and relays. In this task, the NI 9474 digital output module is engaged for triggering signal for fuel injector and spark plug. Each module is provided with transient overvolt protection between other module in the system, the chassis or any connected computer system. Each module is provided with a screw terminal connector as well as backshell for creating custom cable assemblies. The Fig. 2 shows assembled view of NI cRIO 9074 embedded micro controller with its associated I/O modules.

\section{Software Architecture}

In an effort to develop a cost effective RECU a user friendly, virtual instrumentation (VI) based software was used in this work. The functions of the tool are organized in a menu driven structure. The front panel and block diagram are the two graphic user interface of a model or programme to be developed for the task. The user can build the front panel using controls and indicators, which are the interactive input and output terminals of the VI respectively. The block diagram code uses graphical representations of mathematical function to control the front panel objects. The function tool accepts engine parameters and operating data through on screen input and specific data block tile. Wires connect control and indicator terminals contained in the control and simulation tool kit. Data flows through the wires from control to VIs and functions, and from VIs and functions to indicators. The developed system works as a virtual system simulating the operating parameters and displaying all output results graphically and numerically as required. For developing VI system, a graphical programme based software labview version 8.5 and a hardware named compactRIO(cRIO) the products of NI were used. VI not only used for programme development, data analysis, documentation of the results and but also serves as supporting software for hardware functioning.

\section{Development of embedded system}

The embedded system comprise of test engine, hardware and application software. When automotive engines are engaged for testing the developed embedded control system it would be an approach to duplicating the factory made ECU. Further, the control system might operate in accordance with the predesigned parameters of the automotive engine rather than validating the developed one. Hence, single cylinder four stroke 5 HP diesel engine was modified to operate a spark ignited port fuel injection (PFI) engine for embedded system implementation. The preset compression ratio (CR) 17:1 of the engine was modified to CR 10:1 by increasing the clearance volume in the engine head. Diesel injection system was removed from the engine and in the place of diesel pump, a dummy flange was mounted to stop the oil spillage from the engine crankcase. A spark plug was fitted in the place of diesel injector and it is connected with NI 9474 digital 
output module for spark timing and ignition of the engine. The engine head is drilled with two holes of size M14 and M10 for mounting gasoline injector and combustion pressure sensor. The modified engine becomes a baseline engine for the developed RECU and it would be programmed to interface any automobile engine for revealing its performance.

A crank angle encoder is mounted in the camshaft by a specially designed attachment. For every revolution, the encoder develops one pulse and this pulse would be taken as reference signal for triggering signal to spark timing and fuel injection. A MIKUNI make butterfly valve and its associated components of Throttle Position sensor (TPS) and Manifold Absolute Sensor (MAP) assembly was used as intake manifold system. A VI based NI-measurement and automation explorer software was used for developing real time fuel injection control and online sensor data acquisition programmes as shown in the Fig. 3. The measurement and automation software interfaces the VI programme and hardware devices as it has been loaded with drivers for the activation required. The sensors, crank angle encoder, spark plug and fuel injector are connected with the developed RECU system.

\section{Programming throttle position}

The Throttle position sensor (TPS) is mounted on the throttle body and converts the throttle valve angle into an electrical signal. As the throttle opens, the signal voltage increases. At idle, voltage is approximately 0.6 volts on the signal wire. From this voltage, the RECU knows the throttle plate is closed. At wide-open throttle, signal voltage is approximately 3.6 volts. Inside the TPS a resistor and a wiper arm is available and arm is always contacting the resistor. At the point of contact, the available voltage is the signal voltage and this indicates throttle valve position. At idle, the resistance between the power to ground terminal is high, therefore, the available voltage is approximately 0.6. As the contact arm moves closer, the power voltage resistance decreases and the voltage signal increases. Using VI, programme was developed while simulating functions of TPS by observing idle and wide open throttle as minimum and maximum conditions. When the throttle is operated at an intermediate value other than the calibrated one, the TPS - VI programme would give proportional output as it was programmed to follow the linear characteristics of the TPS. Tab. 1 shows the calibrated values of TPS.

Tab. 1. Calibrated Values of Throttle Position Sensor

\begin{tabular}{|c|c|c|c|c|c|}
\hline Throttle Angle $\theta$ & Idle & 20 & 40 & 60 & 80 \\
\hline Voltage Drop, $\mathrm{E}_{\mathrm{o}}$ & 0.6 & 0.8 & 1.6 & 2.4 & 3.2 \\
\hline
\end{tabular}

$$
\begin{gathered}
\mathrm{E}_{\mathrm{o}}=\mathrm{k}_{\mathrm{p}} \Theta \\
\text { when } \Theta=90^{\circ}, \\
\mathrm{E}_{\mathrm{m}}=3.6 \text { Volts, }
\end{gathered}
$$

where:

$\mathrm{E}_{\mathrm{o}}$ - Output voltage, Volts,

$\mathrm{k}_{\mathrm{p}}$ - Sensitivity of potentiometer, $0.04 \mathrm{Volt} /$ degree,

$\mathrm{E}_{\mathrm{m}}$ - Maximum output voltage, Volts.

\section{Programming manifold absolute position}

Intake manifold pressure is a directly related to engine load. The ECU needs to know intake manifold pressure to calculate how much fuel to inject, when to ignite the cylinder, and other functions. The MAP sensor can be located either directly on the intake manifold or it is mounted high in the engine compartment and connected to the intake manifold with vacuum hose. It is critical that the vacuum hose should not have any leaks for proper operation. In this task, the MAP sensor is directly mounted on the intake system. The MAP sensor uses a perfect vacuum as a reference 

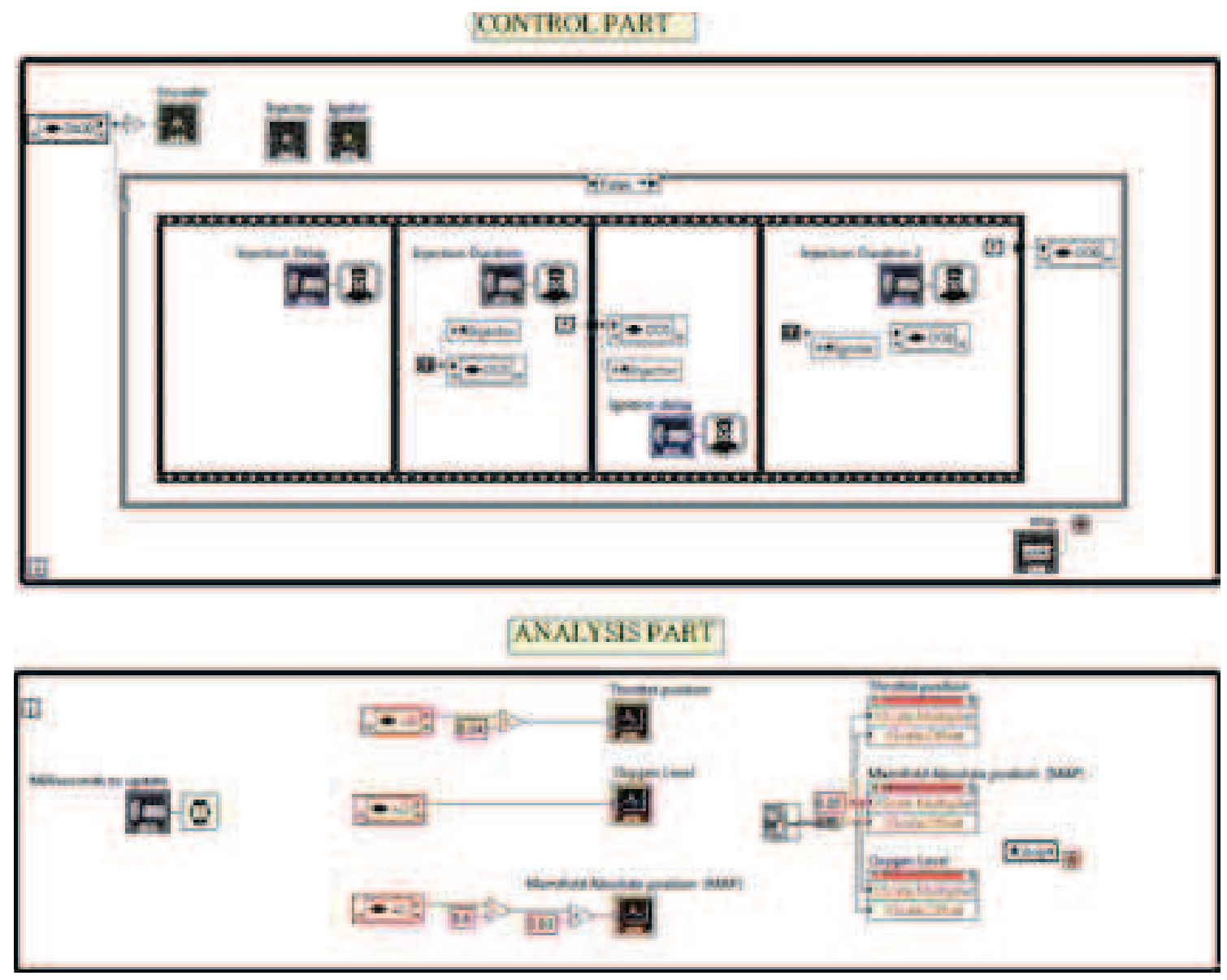

Fig. 3. VI programme for fuel injection control and sensor data acquisition

pressure. The difference in pressure between the vacuum pressure and intake manifold pressure changes the voltage signal. The MAP sensor converts the intake manifold pressure into a voltage signal. Tab. 2 shows the calibrated values of MAP, which was obtained by simulating the functions of sensor using VI, programme. When the engine is operated at an intermediate load other than the calibrated value, the MAP - VI programme would give proportional output as it was programmed to follow the linear characteristics of the MAP.

Tab. 2. Calibrated Values of Manifold Absolute Pressure Sensor

\begin{tabular}{|c|c|c|c|c|c|c|c|}
\hline Voltage Drop $\mathrm{V}_{\mathrm{o}}$ & $0.3-0.5$ & $0.7-0.9$ & $1.1-1.3$ & $1.5-1.7$ & $1.9-2.1$ & $3.2-3.5$ & $3.6-3.8$ \\
\hline Applied Vacuum kPa & 13.3 & 26.7 & 40.0 & 53.5 & 66.7 & 87.2 & 102.7 \\
\hline
\end{tabular}

$$
\mathrm{V}_{\mathrm{o}}=0.03 \mathrm{P}+0.6
$$

when $\mathrm{P}=120 \mathrm{kPa}$,

$$
\begin{gathered}
\mathrm{V}_{\mathrm{o}}=(0.03 \times 120)+0.6 \\
\mathrm{~V}_{\mathrm{m}}=4.2 \text { volts },
\end{gathered}
$$

where:

$\mathrm{V}_{\mathrm{o}}$ - Output voltage from MAP sensor, Volts,

$\mathrm{P}$ - Atmospheric pressure, $\mathrm{kPa}$,

$\mathrm{V}_{\mathrm{m}}$ - Maximum output voltage, Volts.

\section{Programming oxygen sensor}

The RECU uses an oxygen sensor to ensure the air fuel ratio is correct for the catalytic converter. Based on the oxygen sensor signal, the RECU is programmed to adjust the amount of 
fuel injected into the intake air stream. The oxygen sensor generates a voltage signal based on the amount of oxygen in the exhaust compared to the atmospheric oxygen. When exhaust oxygen content is high, oxygen sensor voltage output is low. When exhaust oxygen content is low, oxygen sensor voltage output is high. The greater the difference in oxygen content between the exhaust stream and atmosphere, the higher the voltage signal. From the oxygen content, the RECU could determine if the air fuel ratio is rich or lean by giving output signals as shown in the Tab. 3. A rich mixture consumes nearly all the oxygen, so the voltage signal is high, in the range of 0.6-1.0 volts. A lean mixture has more available oxygen after combustion than a rich mixture, so the voltage signal is low $0.4-0.1$ volts. At the stoichiometric air fuel ratio $(14,7: 1)$ oxygen sensor voltage output is approximately 0.45 volts. When the engine is operated at specified loads oxygen sensor VI programme would give definite output that could indicate the status of the exhaust gas.

Tab. 3. Calibrated value of Oxygen Sensor

\begin{tabular}{|c|c|c|}
\hline Exhaust oxygen Content & Oxygen Sensor Output & Air/Fuel Ratio \\
\hline Low & High, Above 0.45 Volts & Rich \\
\hline High & Low, Below 0.45 volts & Lean \\
\hline
\end{tabular}

\section{Fuel injection mapping}

The amount of fuel injected depends on fuel system pressure and the length of time the injector is turned on. Fuel system pressure is controlled by the pressure regulator, and injector on time is controlled by the RECU. The time the injector is on is often called as duration or pulse width and it is measured in milliseconds (ms). Injector flow rate can be determined based on an effective real world injector operating pulse time and fuel flow. It is unrealistic to establish the fuel flow to the engine based on an injector operating pulse time of $100 \%$ - wide open all the time. Duty cycle is an injector on time that could be understood by the application software. The formula 3 uses an injector operating duty cycle based on $80 \%$. Some full race engine management system may operate at $85-90 \%$ duty cycle, but doing so for the likelihood of overheating the injector, which may cause irregular fuel rates or a decrease in low speed operation.

$$
\text { Injector flow rate }(\mathrm{cc} / \mathrm{min})=\frac{(\text { Engine HP } \mathrm{x} \text { BSFC) }}{\text { (Number of injectors } \mathrm{x} \text { duty cycless) }} \text {, }
$$

where BSFC - Brake specific fuel consumption.

Modern gasoline injection systems involve various sensors fitted in the engine, an electronic control unit (ECU) and mostly solenoids operated fuel injectors to meter and inject the right quantity of fuel into each cylinder of the engine. The ECU receives electrical signals in the form of a current or voltage from various sensors for monitoring different operating engine variables. Depending upon the engine operating variables, the quantity of fuel will be calculated by the ECU and the signal to the injector to inject the fuel will be sent. In order to evaluate the proper quantity of fuel to be injected, it is very much essential to know the characteristics of the fuel injector under the influence of different parameters controlling it. The characteristics of the fuel injectors are different under static and dynamic conditions. Static characteristics can be found out by a bench test conducted using fuel injectors and a control unit, whereas dynamic characteristics can be found out only in running conditions. Also, for these injectors the 'dead time' is an important parameter, which tells about the delay of injector to inject the fuel after receiving a signal. During this period, there is no injection of fuel from the injector. The quantity of fuel injected depends upon a number of parameters like engine speed, fuel pressure, injection duration, injector supply voltage and dead time etc. Effect of the above parameters on the quantity of fuel to be injected gives the injection characteristics of the gasoline injector. 


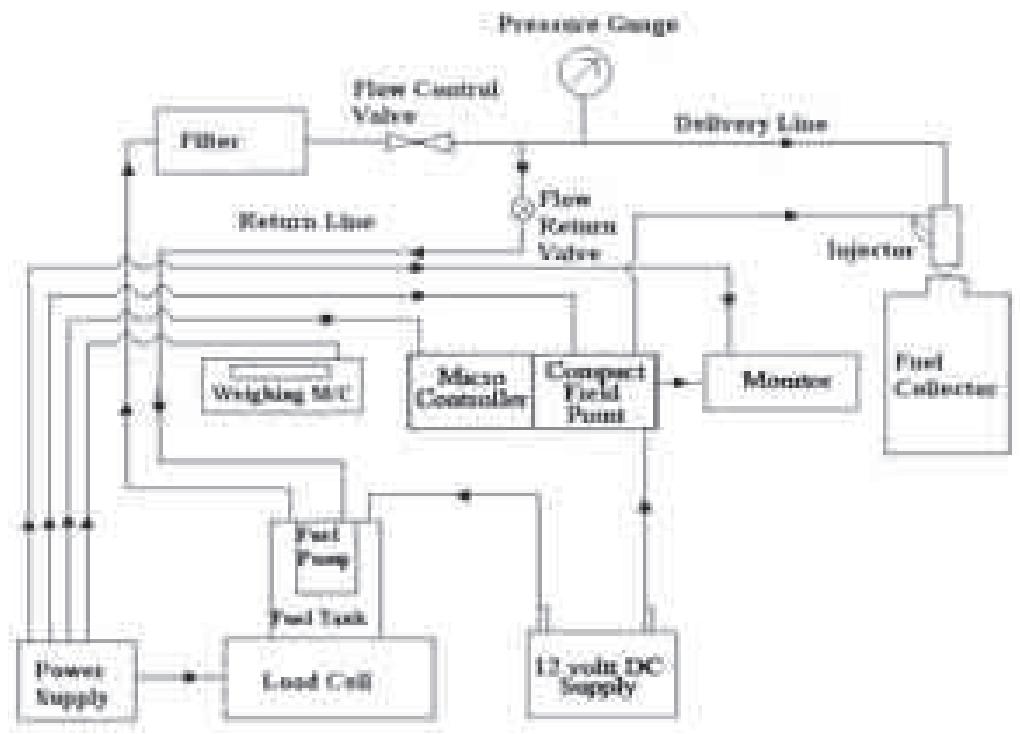

Fig.4. Fuel injection test bench

Figure 4 shows the schematic layout of the test bench setup for the injector test, which consists of fuel tank, in-tank electric fuel pump, electronic fuel injector, electronic control circuit, injection chamber and a hardware named compact field point. In the dynamic injection test, the amount of fuel injected, dynamic, is determined by measuring the injected fuel quantity for 60 seconds at different fuel pressures, injection durations and injector supply voltages. During the dynamic test, the fuel pressure was varied from 0.5 to 5.0 bar in steps of 0.5 bar, injection duration was varied from 2.0 to $20 \mathrm{~ms}$ in steps of $2 \mathrm{~ms}$. The dynamic fuel injection quantity is measured in milligrams per injection. The injected fuel was collected in an injection chamber and the difference between initial and final weights was measured using an electronic weighing machine. The Fig. 5 shows fuel injection quantity corresponding to various pulse width when the injector was operated at different pressures. In this study, experiments were conducted with two types of injectors commonly available in the market, namely, single-hole and multi hole injectors. The diameter of the orifice of the single hole injector is $0.5 \mathrm{~mm}$ and the diameter of each orifice of the multi-hole injector is $0.225 \mathrm{~mm}$. The injector is fixed on the injection chamber by a specially fabricated fuel rail and injector holder to fix one injector only. The injection dead time is influenced by fuel pressure and injector supply voltage. The developed VI programme for fuel injection and its measurement is shown in Fig. 6 and 7 respectively.

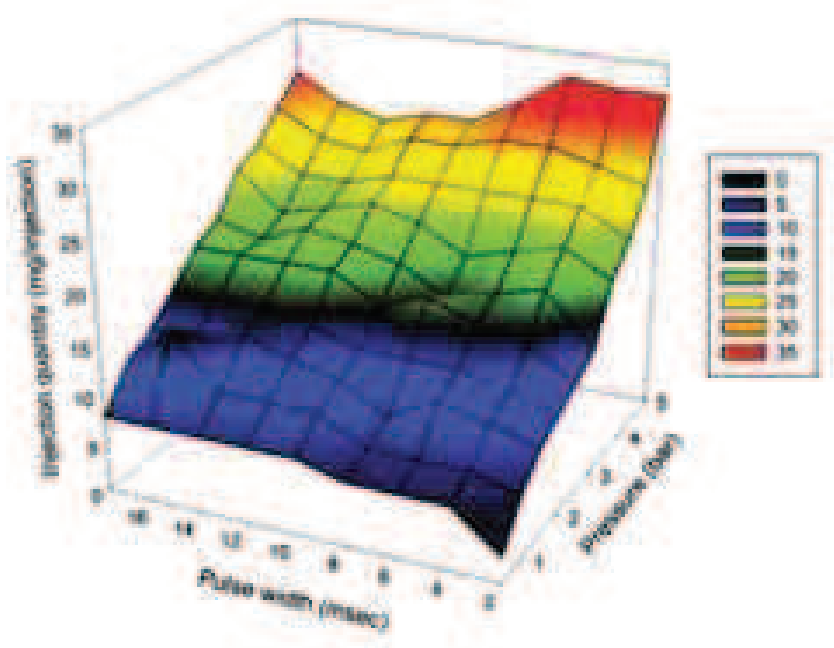

Fig.5. Pulse width measurement 


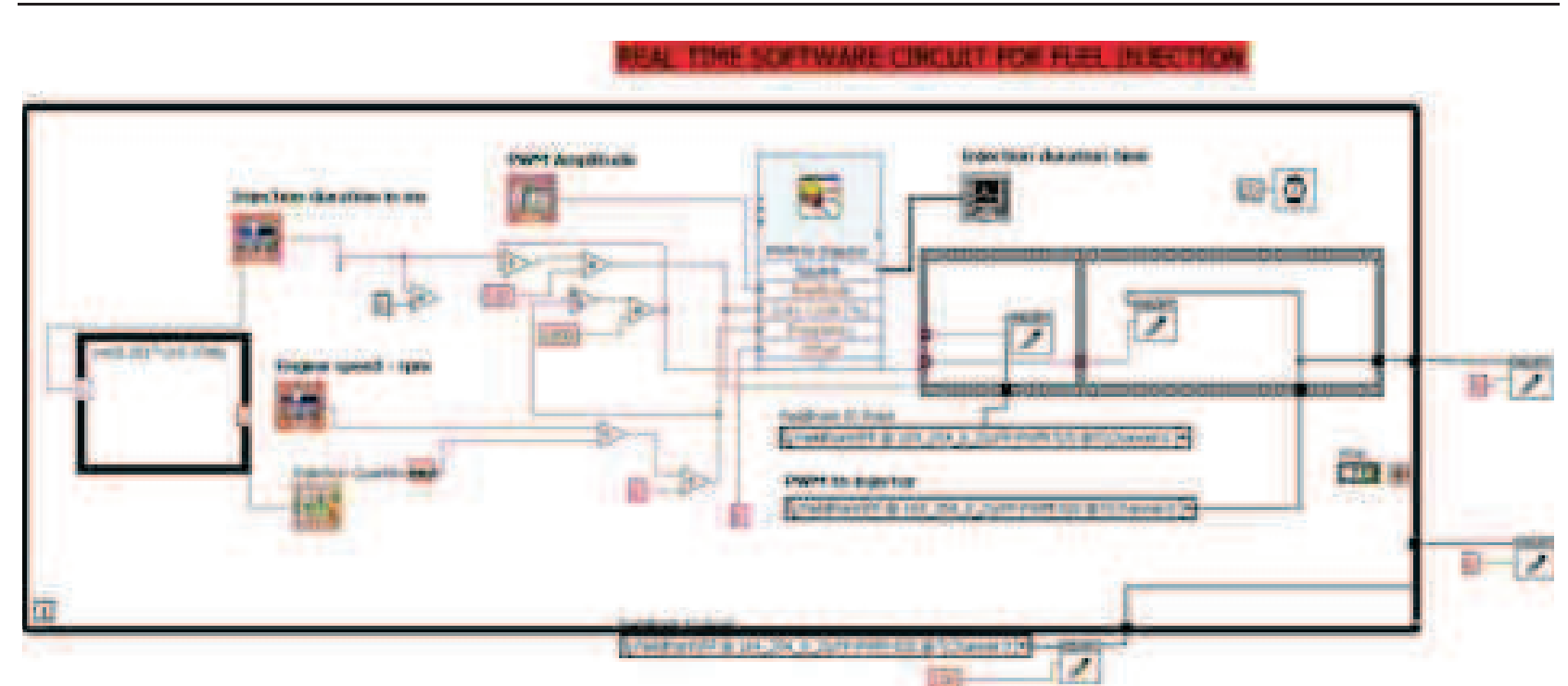

Fig. 6. Virtual instrumentation programme (Bolck diagram) for fuel injection and measurement

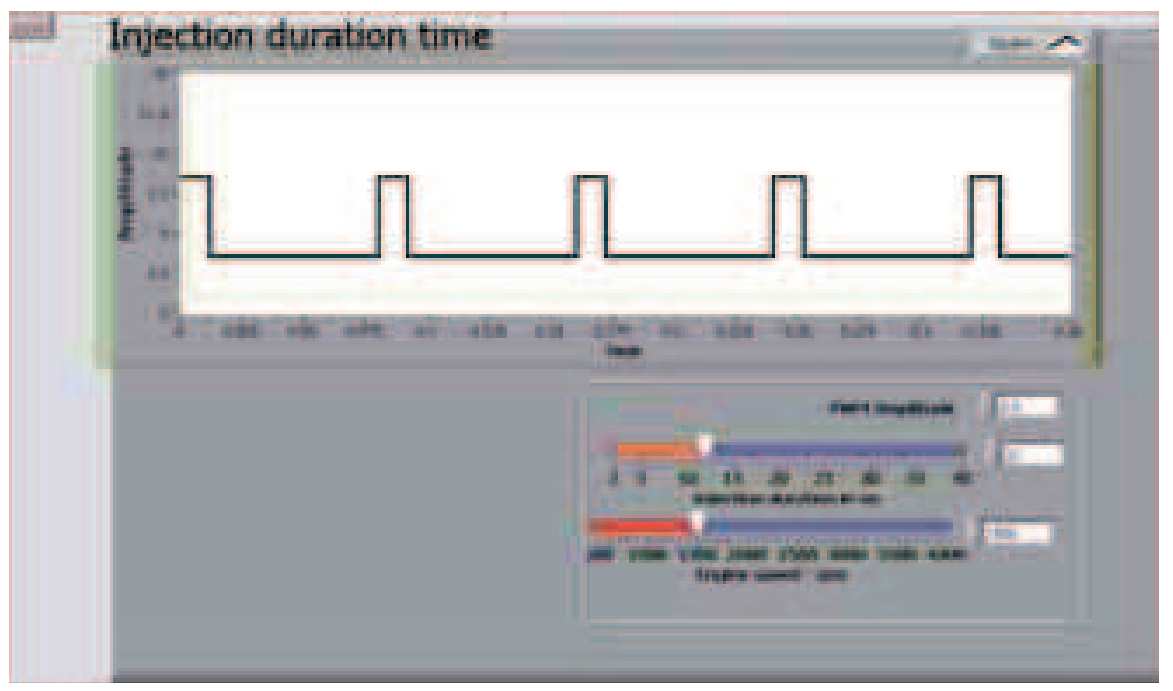

Fig. 7. Virtual instrumentation programme (Front panel) for pulse width measurement

The injection dead time is calculated using the dynamic fuel injection quantity at the point where the fuel injection quantity starts to vary linearly with the injection duration. In this study, the dynamic fuel injection quantity starts to vary linearly at an injection duration of $2 \mathrm{~ms}$. So, the injection time is calculated using the dynamic fuel injection quantity at the injection duration of $2 \mathrm{~ms}$. The effect of injection dead time is much in the case of dynamic fuel injection quantity since the total injection duration and injection dead time are in the order of milliseconds. But, in the case of static fuel injection rate, the effect of injection dead time is negligible, because the total injection duration is in the order of seconds whereas the injection dead time is in the order of milliseconds.

\section{Engine control}

In order to provide the correct amount of fuel for every operating condition, the engine control unit (ECU) has to monitor a huge number of sensor inputs. The duty cycle of an automotive engine could be determined by performing tests at engine test bed accommodated with ECU, necessary sensors and actuators. With the use of RECU, the duty cycle for the required operation could be estimated by conduction test on a tabletop - fuel injection test bench while simulating the functions of the sensors using VI programme. 
The ECU uses a formula and large number of lookup tables to determine the pulse width for given operating conditions. The equation would be a series of many factors multiplied by each other. Many of these factors would come from lookup tables. In this task, the equation involves only three factors, whereas an automotive application might have more than hundred parameters each with its own lookup table.

$$
\begin{aligned}
\text { Pulse width } & =\left(\mathrm{T}_{\mathrm{b}}\right) \times(\text { Factor } \mathrm{A}) \times(\text { Factor } \mathrm{B}), \\
T_{b} & =\frac{M A F}{r(N / 2)(A / F)},
\end{aligned}
$$

where:

$\mathrm{T}_{\mathrm{b}} \quad$ - base pulse width,

MAF - Mass air flow rate $\mathrm{kg} / \mathrm{sec}$,

$\mathrm{r} \quad-$ Speed of the engine,

$\mathrm{N} \quad-$ Number of cylinders,

$(\mathrm{A} / \mathrm{F})_{\mathrm{R}}-$ Required air fuel ratio.

In order to calculate the pulse width, the RECU looks up the base pulse width in a look up table. Base pulse width is a function of engine speed and load, which could be calculated from MAP signal as shown in the formula 5. The overall pulse width could be determined by multiplying series of factors loaded in a separate lookup tables. Since the task is open loop, the engine was operated at specific load conditions only. At operating points while the hardware acquiring data, VI programme would make computations for determining appropriate pulse width as the embedded system has been calibrated earlier.

Table 4. Calibrated values for engine control system

\begin{tabular}{|c|c|c|}
\hline $\begin{array}{c}\text { Base pulse } \\
\text { width }\end{array}$ & A & Factor A \\
\hline \multirow{4}{*}{$\mathrm{T}_{\mathrm{b}}$} & 20 & 1.2 \\
\cline { 2 - 3 } & 40 & 1.1 \\
\cline { 2 - 3 } & 60 & 1.0 \\
\cline { 2 - 3 } & 80 & 0.9 \\
\hline
\end{tabular}

\begin{tabular}{|c|c|}
\hline B & Factor B \\
\hline 0 & 2.0 \\
\hline 1 & 1.8 \\
\hline 2 & 1.4 \\
\hline 3 & 1.0 \\
\hline 4 & 0.7 \\
\hline
\end{tabular}

Factor A- Proportional pulse width for MAP sensor

Factor B - Proportional pulse width for TPS sensor

In the final phase, the developed programme was deployed in NI 9074 real time microcontroller and RECU was interfaced with the test engine. Using labview FPGA, real-time control strategy was implemented for fuel injection and spark timing. The engine was aimed to operate at stoichiometric air fuel ratio as the RECU has been programmed and calibrated for that strategy. It was observed that at all operating points while the real time pulse width matches with the calibrated value the oxygen level could not meet the desired value. A marginal loss of engine power was also observed, as the estimated pulse width was insufficient to develop required torque. The possible reason is while developing VI programme correction coefficients, time delay and transient effects were not considered to compensate losses. For validation purpose, pulse width was manually adjusted to read the torque and oxygen sensor output at desired value. At all operating load, $10-12 \%$ loss of engine power was recorded which could be correlated with the correction coefficients of various parameter.

\section{Conclusion}

The RECU developed on virtual instrumentation based software could eliminate writing of tens of thousands of text codes while developing programme for measurement applications. With the simulated facility, RECU enhance the developer to confine most of the operating parameters of 
engine away from real test environment. The RECU was achieving performance comparable to the factory ECU while providing the ability to carry out control algorithm research and development, which is not possible with production - oriented electronics. The reconfigurable compactRIO enables the RECU to be engaged for variety automotive testing and measurement applications especially embedded prototyping.

\section{Acknowledgments}

We would like to thank Mr. S. Prasnna, Mr. V. Arul and Mr. K. Siddarth, Trident Tech Labs NI Products, Chennai division, for the support and use of their facility. We would also like to thank Mr. K. Kumaravel, Engine Research Laboratory, Annamalai University and Mr. M. Varun, Anna University, Chennai for their support and encouragement. Their help and enthusiasm for this project has greatly contributed to the initial success of the project.

\section{References}

[1] Dase, C., Sullivan Falcon, J., Maccleery, B., Motorcycle Control Prototyping Using an FPGA-Based Embedded Control System, IEEE Control System Magazine, Vol. 26, No. 5, pp. 17-21, 2006.

[2] Drivven, Inc. Homeapage, Driven-Powertrain Rapid Control Prototyping, Available: http://www.driven.com.

[3] National Instruments Labview Real-Time Web portal, Labview Real-Time for Measurement and Control, Available: http://www.ni.com/realtime.

[4] Xilinx product page, System Generator for DSP - the Leading - Edge Modeling and Implementation tool for High Performance DSP system, Available: http//www.xilinx.com./ ise/options_prod/system_generator.htm.

[5] Celoxica homepage, Welcome to Celoxica - the Technology Leader in C Based Design and Synthesis, http://www.celoxica.com.

[6] National Instruments Labview Toolkit Web portal, Labview Toolkits, Available: http//www.ni.com/tookits.

[7] National Instruments Labview FPGA Web portal, Labview FPGA - Customize Your Hardware Without Having to Build it, Available: http://www.ni.com /fpga. 